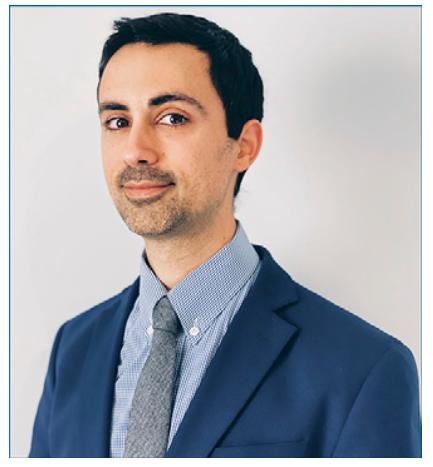

DANIEL L. HALL, PhD

Daniel L. Hall, PhD, is a licensed clinical health psychologist at Massachusetts General Hospital and an Assistant Professor of Psychology at Harvard Medical School. $\mathrm{He}$ is a faculty member of the Mongan Institute Health Policy Research Center and a core member of the MGH Behavioral Medicine Program, Benson-Henry Institute, Cancer Outcomes Research and Education (CORE) Program, and Cancer Survivorship Program. Dr. Hall's interests include uncertainty, stress, and health behaviors among adults affected by cancer, and the development and implementation of behavioral interventions for managing these concerns. His research is currently supported by the National Institutes of Health and the American Cancer Society. You can follow him on Twitter at @DanielHallPhD.

doi: $10.6004 /$ jnccn.2020.7682

The ideas and viewpoints expressed in this commentary are those of the author and do not necessarily represent any policy, position, or program of NCCN.

\section{Wake Up Oncologists! It's Time to Help Our Patients With Insomnia}

\author{
Daniel L. Hall, PhD, and Jeffrey M. Peppercorn, MD, MPH
}

$I^{n}$ nsomnia is among the most prevalent and debilitating problems faced by cancer survivors, affecting $30 \%$ to $60 \%$ of adults diagnosed with cancer. ${ }^{1}$ Having insomnia places patients with cancer at greater risk of impaired mental health (ie, major depression, generalized anxiety) and physical health (ie, fatigue, poorer immune functioning). ${ }^{2}$ The NCCN Clinical Practice Guidelines in Oncology (NCCN Guidelines) for Survivorship recommend routine screening for sleep difficulties among all patients. ${ }^{3}$ These guidelines propose 3 brief questions for assessing sleep, suggest evaluation of contributing factors (eg, comorbidity, medications, shift work), and identify multiple options for evidence-based therapy. However, unlike disease management guidelines, there is little evidence that these recommendations for insomnia are embraced in practice. It is past time for oncology clinicians to wake up to the scope of this problem, the ease of screening, and the availability of effective options to treat insomnia among our patients.

Although a growing body of literature has demonstrated the high prevalence and impact of insomnia among patients with cancer, insomnia is a not a focus of oncology training and it is not consistently addressed in practice. In this context, Harrold et al ${ }^{4}$ investigated the prevalence of sleep disturbance in a large sample of patients with varying cancer types presenting for routine care at a hospital-based oncology clinic in Ireland, reporting their findings elsewhere in this issue. They found that nearly two-thirds of cancer survivors experienced sleep disturbance, and an alarming 1 in 3 met criteria for insomnia syndrome. In addition to serving as a call to action for Ireland's oncology clinics, these high prevalence rates are similar to estimates among cancer survivors in the Unites States and support the recognition of sleep disturbance as a universal issue. Undoubtedly, in the time since these data were collected, additional stressors experienced by cancer survivors during the COVID-19 global pandemic have only exacerbated high rates of sleep disturbance. This report also identifies factors associated with a greater likelihood of insomnia (eg, breast cancer history, age $<65$ years, use of alcohol as a sleep aid), although some clinically meaningful details were notably absent (eg, insomnia duration of 1 month vs 3 months, cancer staging, more granular age strata below 65 years). Collectively, the findings by Harrold et $\mathrm{al}^{4}$ underscore the critical need for routine implementation of insomnia screening and greater access to evidence-based insomnia treatment within cancer clinics globally.

So, what is getting in the way of routine insomnia screening? Perhaps oncology practice has not recognized the strong evidence base supporting behavioral medicine and other nonpharmacologic approaches to address this common problem. Only approximately half (56\%) of US cancer centers report any systematic screening for insomnia, among which only 1 in every 4 patients is screened. ${ }^{5}$ Similarly, Harrold et al ${ }^{4}$ found that only 1 in 3 (34\%) cancer survivors in Ireland recalled being asked about sleep during a cancer-related clinical encounter. One of the major challenges may be a misperception that a specialist is required to identify sleep problems and offer treatment referrals. Among 25 NCCN Member Institutions surveyed regarding management of insomnia, most (72\%) identified lack of

See page 1623 for related article. 
specialists as a chief barrier to addressing insomnia. ${ }^{5}$ Although a specialist may be required for diagnosing certain conditions (eg, obstructive sleep apnea), the NCCN Guidelines make it clear that oncology clinicians should at a minimum assess the presence of sleep difficulties. Patients may be hesitant to bring up sleep difficulties unless prompted, and therefore oncology clinicians need to be proactive about assessing sleep. To make this easy, NCCN Guidelines ${ }^{3}$ include 3 simple questions to ask patients at regular intervals (Table 1 ) as well as a decision tree to guide management, including referrals as appropriate.

If successful, increased screening by oncology clinicians will result in identifying more patients who stand to benefit from gold standard treatments for insomnia. Oncology clinicians may not be aware that cognitive behavioral therapy for insomnia (CBT-I) is highly efficacious for treating insomnia among cancer survivors and is recommended by the American College of Physicians as the first-line treatment. ${ }^{6}$ CBT-I teaches skills for sleep hygiene, stimulus control, modifying sleep deprivation (eg, sleep restriction), reframing unhelpful beliefs about sleep, and promoting relaxation. Compared with pharmacotherapy, the effects of CBT-I are more durable, because patients learn skills to address current and future insomnia episodes. Among adults with cancer and insomnia, CBT-I improves sleep outcomes, with moderate-to-large clinical improvements after 4 to 10 sessions that typically persist for a year or longer. ${ }^{7}$ For all its benefits, CBT-I can be a demanding treatment. Patients may be instructed to adhere to (structured) acute sleep deprivation, adjust longstanding bedtime behaviors such as nighttime eating, and confront maladaptive beliefs about sleep. To buffer against high rates of attrition from CBT-I, clinicians typically offer patient-centered modifications tailored to patients' preferences, the severity of insomnia symptoms, and specific insomnia-perpetuating factors (eg, late effects of cancer treatment affecting sleep).

There remain critical questions about how to make evidence-based, nonpharmacologic interventions more accessible. CBT-I is traditionally delivered inperson, which is not feasible for many patients with cancer, particularly given social distancing requirements due to the present COVID-19 global pandemic. For patients with cancer, barriers to CBT-I include time limitations, travel, and illness burden constraints. Access and delivery can be made easier through use of innovative methods, several of which are highlighted in the report by Harrold et al, ${ }^{4}$ chiefly asynchronous options for remote delivery by telephone, videoconferencing, website, and smartphone apps. Many of those formats have been proven to be efficacious in studies of adults affected by cancer ${ }^{8}$ and have comparable effects on sleep outcomes. ${ }^{7,8}$ However, patients with cancer may be less engaged in treatment through remote delivery that does not include real-time reinforcement from a CBT-I trained therapist. Synchronous telehealth delivery of CBT-I offers the psychological benefits of face-to-face, real-time communication while addressing common physical and logistical barriers to delivering in-person CBT-I in routine clinical oncology practice. In the United States, Medicaid has approved telemedicine reimbursement in 48 states, ${ }^{9}$ a critical reform that can make it feasible for cancer centers to hire CBT-I therapists to provide synchronous, remotely delivered insomnia

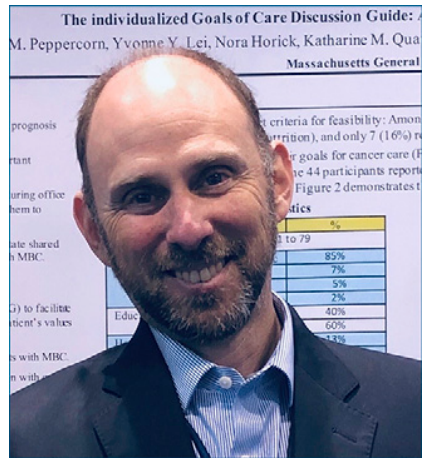

JEFFREY M. PEPPERCORN, MD, MPH

Jeffrey M. Peppercorn, MD, MPH, is a medical oncologist specializing in breast cancer who conducts research related to health policy, bioethics, and cancer survivorship. He serves as the Director of Supportive Care and Survivorship for the Massachusetts General Hospital Cancer Center and is an Associate Professor of Medicine at Harvard Medical School. He is an Associate Editor for JNCCN-Journal of the National Comprehensive Cancer Network, and has served on the NCCN Survivorship Guidelines Committee and the Sleep Disorders Survivorship Subcommittee and contributed to current NCCN insomnia guidelines. He has published more than 150 papers and chapters on issues related to survivorship, bioethics, health policy, and breast cancer care. You can follow him on Twitter at @ethicsdoctorP. 
treatment to patients. Finally, oncology clinicians should assess patients' preferences for other evidence-based, nonpharmacological insomnia treatments (eg, acupuncture, yoga, physical activity) and offer referrals to trained providers in their cancer center or surrounding community. ${ }^{10,11}$

The impact of insomnia can be subtle, and the condition is also common in the general population; thus, oncologists may not address this problem with the same diligence applied to other aspects of cancer care. We look forward to clinical oncology recognizing the needs and preferences of cancer survivors with regard to insomnia treatment. The findings reported by Harrold et $\mathrm{al}^{4}$ underscore the near-ubiquity of sleep disturbance among patients with cancer and, importantly, their interest in accessing insomnia treatment. The question for us in clinic tomorrow is not whether many of our patients are getting poor sleep and want help, but rather when are we going to start asking about this issue and trying to address it. Ultimately, the NCCN Guidelines for sleep screening are only as good as our education of clinicians and implementation in practice. We implore clinical teams to consider these guidelines as a primary obligation, on par with appropriate management of the cancer.

To this end, methods to facilitate dissemination and intervention are of paramount importance. The need to implement routine screening and treatment of sleep difficulties in patients with cancer is clear. In addition to the delivery methods discussed earlier, stepped-care approaches could also be considered, whereby patients are offered asynchronous content (such as a prerecorded educational video) or a group educational session, followed by reassessment and triage to more intensive intervention and follow-up clinical visits as needed. In this regard, we commend Harrold et $\mathrm{al}^{4}$ on their plans to develop an educational pamphlet for patients. We trust that this will address sleep hygiene, suggest options for therapy, and encourage patients to advocate for attention to their insomnia at their oncology visits. Acknowledging the challenges to screening uptake within cancer centers, a similar pamphlet for oncology clinicians is likely needed as well. Together with support from their institutions, oncology clinicians and supportive care providers are well positioned to implement NCCN Guidelines on sleep assessment and treatment. There has never been a more critical time to help our patients get the rest they so badly need.

Disclosures: Dr. Hall has disclosed that he has no financial interests, arrangements, or affiliations with the manufacturers of any products discussed in this article or their competitors. Dr. Peppercorn has disclosed that he receives consulting fees from Athenex and Abbott Labs, and that his spouse is employed by GlaxoSmithKline.

Correspondence: Daniel L. Hall, PhD, Harvard Medical School, 100 Cambridge Street, 16th floor, Boston, MA 02114

Email: dhall7@mgh.harvard.edu

\section{References}

1. Savard J, Ivers H, Villa J, et al. Natural course of insomnia comorbid with cancer: an 18-month longitudinal study. J Clin Oncol 2011;29:3580-3586.

2. Ancoli-Israel S. Recognition and treatment of sleep disturbances in cancer. J Clin Oncol 2009;27:5864-5866.

3. Denlinger CS, Sanft T, Moslehi JJ, et al. NCCN Guidelines Insights: Survivorship, Version 2.2020: featured updates to the NCCN Guidelines. J Natl Compr Canc Netw 2020;18:1016-1023.

4. Harrold EC, Idris F, Keegan NM, et al. Prevalence of insomnia in an oncology patient population: an Irish tertiary referral center experience. J Natl Compr Canc Netw 2020;18:1623-1630.

5. Zhou ES, Partridge AH, Syrjala KL, et al. Evaluation and treatment of insomnia in adult cancer survivorship programs. J Cancer Surviv, 2017;11:74-79.

6. Qaseem A, Kansagara D, Forciea MA, et al. Management of chronic insomnia disorder in adults: a clinical practice guideline from the American College of Physicians. Ann Intern Med 2016;165:125-133.
7. Ma Y, Hall DL, Ngo LH, et al. Efficacy of cognitive behavioral therapy for insomnia in breast cancer: a meta-analysis. Sleep Med Rev 2020;55: 101376.

8. Johnson JA, Rash JA, Campbell TS, et al. A systematic review and metaanalysis of randomized controlled trials of cognitive behavior therapy for insomnia (CBT-I) in cancer survivors. Sleep Med Rev 2016;27:20-28.

9. Mehrotra A, Huskamp HA, Souza J, et al. Rapid growth in mental health telemedicine use among rural Medicare beneficiaries, wide variation across states. Health Aff (Millwood) 2017;36:909-917.

10. Garland SN, Xie SX, DuHamel K, et al. Acupuncture versus cognitive behavioral therapy for insomnia in cancer survivors: a randomized clinical trial. J Natl Cancer Inst 2019;111:1323-1331.

11. Kwak A, Jacobs J, Haggett D, et al. Evaluation and management of insomnia in women with breast cancer. Breast Cancer Res Treat 2020;181: 269-277. 